\title{
The ultra-compact binary 4U 1850-087 observed with INTEGRAL: hard X-ray emission from an X-ray burster ${ }^{\star}$
}

\author{
L. Sidoli ${ }^{1}$, A. Paizis ${ }^{1}$, A. Bazzano ${ }^{2}$, and S. Mereghetti ${ }^{1}$ \\ 1 INAF-IASF, Istituto di Astrofisica Spaziale e Fisica Cosmica Milano, via E.Bassini 15, 20133 Milano, Italy \\ e-mail: sidoli@iasf-milano.inaf.it \\ 2 INAF-IASF, Istituto di Astrofisica Spaziale e Fisica Cosmica Roma, via del Fosso del Cavaliere 100, 00133 Roma, Italy \\ Received 5 June 2006 / Accepted 2 September 2006
}

ABSTRACT

\begin{abstract}
Context. The X-ray burster 4U 1850-087, located in the Galactic globular cluster NGC 6712, is an ultracompact binary (orbital period $\sim 21 \mathrm{~min}$ ), likely harbouring a degenerate companion.

Aims. The source has been observed at soft $\gamma$-rays several times with the INTEGRAL satellite, during the monitoring of the Galactic plane, with an unprecedented exposure time. We analysed all available INTEGRAL observations, with the main aim of studying the long-term behaviour of this Galactic bulge X-ray burster.

Methods. The spectral results are based on the systematic analysis of all INTEGRAL observations covering the source position performed between March 2003 and November 2005.

Results. The source X-ray emission is hard and is observed, for the first time, up to $100 \mathrm{keV}$. A broad-band spectrum obtained combining the INTEGRAL spectrum together with a quasi-simultaneous XMM-Newton observation performed in September 2003 is well modeled with a disk-blackbody emission (with an inner disk temperature of $\sim 0.8 \mathrm{keV}$ ) together with a power-law (with a photon index, $\Gamma$, of 2.1). The $2-100 \mathrm{keV}$ luminosity is $1.5 \times 10^{36} \mathrm{erg} \mathrm{s}^{-1}$ (assuming a distance of $6.8 \mathrm{kpc}$ ).

Conclusions. INTEGRAL observations reveal for the first time that this X-ray burster displays a very hard X-ray spectrum, with a cut-off at energies higher than $100 \mathrm{keV}$, and that the source spends most of the time in this low luminosity and hard state. Indeed, a previous BeppoSAX observation in April 1997 observed high energy emission from 4U 1850-087 only up to $50 \mathrm{keV}$.
\end{abstract}

Key words. X-rays: stars: individual: 4U 1850-087 - X-rays: binaries

\section{Introduction}

4U 1850-087 (Swank et al. 1976) is an X-ray burster located in the galactic globular cluster NGC 6712. The likely optical counterpart displays an UV modulation (Anderson et al. 1993) with a period of $20.6 \mathrm{~min}$ (interpreted as the orbital period), implying a degenerate companion of $0.04 M_{\odot}$ (Homer et al. 1996).

The first broad-band spectrum from this source was obtained with BeppoSAX in the energy range $0.3-50 \mathrm{keV}$ during a pointing performed in 1997 (Sidoli et al. 2001). The spectrum was well described with a disk-blackbody and Comptonized continuum with $N_{\mathrm{H}}=3.9 \times 10^{21} \mathrm{~cm}^{-2}$, an inner disk temperature, $k T_{\text {in }}$, of $\sim 0.6 \mathrm{keV}$, an inner projected radius of $\sim 5 \mathrm{~km}$ (for an assumed NGC 6712 distance of $6.8 \mathrm{kpc}$, Harris 1996), a temperature, $k T_{0}$, of the input "seed" photons, of $0.8_{-0.2}^{+0.1} \mathrm{keV}$, an electron temperature, $k T_{\mathrm{e}}$, of $70 \mathrm{keV}$, and an optical depth, $\tau$, of 1.7 . The estimated $0.1-100 \mathrm{keV}$ luminosity was $1.9 \times 10^{36} \mathrm{erg} \mathrm{s}^{-1}$ (at $6.8 \mathrm{kpc}$ ).

Other X-ray observations are reported for 4U 1850-087 limited to the energy range below $10 \mathrm{keV}$ ( $A S C A$, Juett et al. 2001; XMM-Newton, Sidoli et al. 2005; Chandra, Juett \& Chakrabarty 2005).

* Based on observations with INTEGRAL, an ESA project with instruments and the science data centre funded by ESA member states (especially the PI countries: Denmark, France, Germany, Italy, Switzerland, Spain), Czech Republic and Poland, and with the participation of Russia and the USA.
We report here the first detection of 4U 1850-087 above $50 \mathrm{keV}$ with the INTEGRAL satellite, obtained with an unprecedented exposure time collected during the monitoring of the Galactic plane.

\section{Observations and results}

The ESA INTEGRAL gamma-ray observatory, launched in October 2002, carries three co-aligned coded mask telescopes: the imager IBIS (Ubertini et al. 2003), which allows high angular resolution imaging over a large field of view $\left(29^{\circ} \times 29^{\circ}\right)$ in the energy range $15 \mathrm{keV}-10 \mathrm{MeV}$, the spectrometer SPI (Vedrenne et al. 2003; $20 \mathrm{keV}-8 \mathrm{MeV}$ ) and the X-ray monitor JEM-X (Lund et al. 2003; 3-35 keV). IBIS is composed of a lowenergy CdTe detector (ISGRI; Lebrun et al. 2003), sensitive in the energy range from $15 \mathrm{keV}$ to $1 \mathrm{MeV}$, and a CsI detector (PICsIT; Labanti et al. 2003), designed for optimal performance at $511 \mathrm{keV}$, and sensitive in the $175 \mathrm{keV}-10 \mathrm{MeV}$ energy range.

We analyzed all public and Core Program IBIS observations pointed within $10^{\circ}$ of the source. This resulted in 909 individual pointings (Science Windows, SWs) performed between March 2003 and November 2005. All the data have been processed using version 5.1 of the OSA INTEGRAL analysis software, and analysed with the corresponding rebinned response matrices.

A mosaic image of all 909 pointings revealed the sources that were active in this field during the period of interest. The list of all the detected sources (detection significance $>5 \sigma$ ) was then used in the spectral step, where spectra for all the detected 
Table 1. Summary of all INTEGRAL observations of 4U 1850-087 analysed here. The observations have been grouped together in 4 datasets for brevity. The 3rd and 4th columns list the Start and Stop Time of the four groups of observations, the fifth column reports the number of SWs in each data-set. Data-set n. 4 includes Core Program data still not publicly available.

\begin{tabular}{lllll}
\hline \hline $\begin{array}{l}\text { Data } \\
\text { set }\end{array}$ & $\begin{array}{l}\text { Temporal } \\
\text { window }\end{array}$ & $\begin{array}{l}\text { Start Time } \\
\text { (MJD) }\end{array}$ & $\begin{array}{l}\text { End Time } \\
\text { (MJD) }\end{array}$ & $\begin{array}{l}\text { Num of } \\
\text { SWs }\end{array}$ \\
\hline 1 & Mar. 2003-May 2003 & 52708.7 & 52772.0 & 258 \\
2 & Sep. 2003-Nov. 2003 & 52910.9 & 52963.4 & 210 \\
3 & Mar. 2004-May 2004 & 53075.1 & 53126.5 & 84 \\
4 & Aug. 2004-Nov. 2005 & 53238.1 & 53684.0 & 357 \\
\hline
\end{tabular}

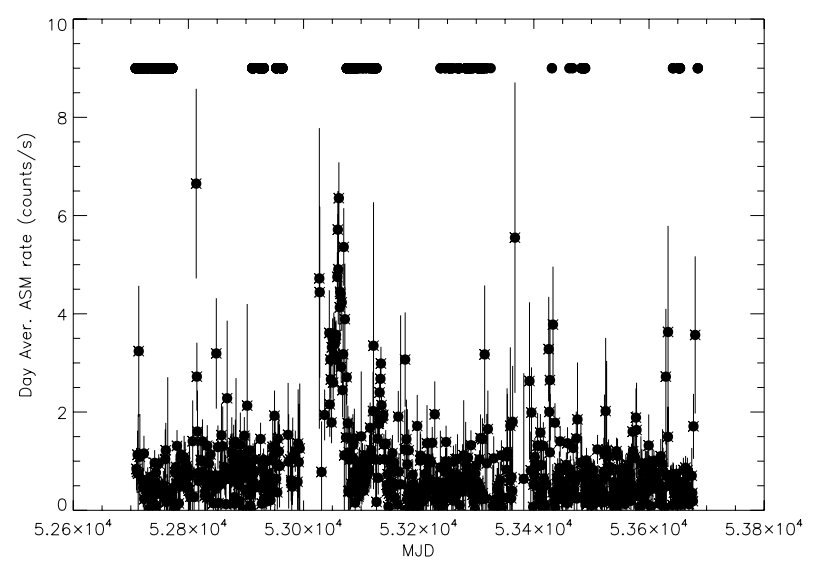

Fig. 1. RXTE/ASM lightcurve (day average) of 4U 1850-087. The small solid circles in the upper region show the times of the INTEGRAL observations analysed here.

sources in the field of view were simultaneously extracted with the standard spectral extraction method.

$4 \mathrm{U} 1850-087$ is a relatively faint source, therefore a meaningful spectral analysis needs to be performed adding together several observations. For this reason, we grouped the pointings in four data-sets (listed in Table 1) from which we extracted four different IBIS/ISGRI spectra.

The 2-10 keV flux of $4 \mathrm{U} 1850-087$ shows a brightening event peaking on 25 February 2004, and lasting $\sim 10$ days, as visible from the ASM/RXTE lightcurve reported in Fig. 1. Only part of its decay is covered by the INTEGRAL observations of data-set 3.

When we are averaging the spectra of $4 \mathrm{U} 1850-087$ using data that span over different mission times, we use a systematics of 5\% in the spectral fit process, to take into account for modifications in the instrument evolution (Lubinski et al. 2005).

The best-fit spectral results, using a single power-law are reported in Table 2 for the four data-sets. The fits display a high $\chi^{2}$ (with a null hypothesis probability in the range $0.1-2.4 \%$ ) which can be explained by the spectral distortions present when extracting IBIS/ISGRI spectra spanning very different mission times (Lubinski et al. 2005), especially for faint sources, and by possible intrinsic source spectral changes on smaller timescales, which cannot be a priori excluded. Nevertheless, the global spectral shape is rather constant on timescales of months-years.

We tried also to extract JEM-X spectra, but the smaller field of view, the faintness of the source, together with some instrumental issues (e.g. JEM-X switched-off in several occasions), severely reduced the number of useful observations. Thus data could be used only for the integrated spectrum covering the dataset 3 and part of the data-set 4 . The JEM-X spectra correspond to
Table 2. Results of the spectral analysis of the four temporal-selected IBIS/ISGRI spectra. Data-set numbers are the same as in Table 1. The 2 nd column reports the net exposure time of each spectrum. The 3 rd column lists the IBIS/ISGRI average rates in the energy range $20-100 \mathrm{keV}$. Fluxes are in units of $10^{-10} \mathrm{erg} \mathrm{cm}^{-2} \mathrm{~s}^{-1}(20-100 \mathrm{keV})$.

\begin{tabular}{lrllll}
\hline \hline Data-set & $\begin{array}{r}\text { Exp. } \\
(\mathrm{ks})\end{array}$ & $\begin{array}{l}\text { IBIS/ISGRI rate } \\
\left(\mathrm{s}^{-1}\right)\end{array}$ & Photon Index & Flux & $\chi^{2} /$ d.o.f. \\
\hline 1 & 372 & $1.25 \pm 0.05$ & $2.5 \pm 0.2$ & 0.8 & $21.6 / 8$ \\
2 & 383 & $2.36 \pm 0.05$ & $2.1 \pm 0.1$ & 1.8 & $18.8 / 7$ \\
3 & 114 & $1.42 \pm 0.09$ & $2.2 \pm 0.3$ & 1.1 & $11.6 / 7$ \\
4 & 490 & $1.59 \pm 0.05$ & $2.2 \pm 0.1$ & 1.2 & $16.1 / 7$ \\
\hline
\end{tabular}

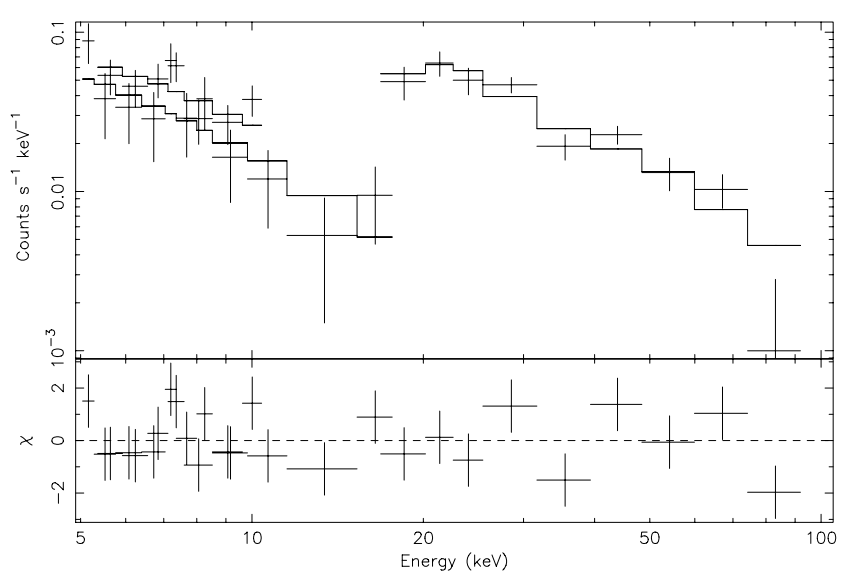

Fig. 2. 4U 1850-087 combined JEM-X and IBIS/ISGRI simultaneous spectrum, fit with a single power-law (photon index of $2.2 \pm 0.2$ ). In the lower panel, the residuals in units of standard deviations are shown.

$76.7 \mathrm{ks}$ net exposure time for JEM-X1 and $55.5 \mathrm{ks}$ for JEM-X2, with a source count rate of $0.23 \pm 0.02$ and $0.22 \pm 0.03$, respectively in the two JEM-X units $(5-20 \mathrm{keV})$.

The combined JEM-X plus IBIS/ISGRI simultaneous spectrum (taken from the data-set 3) is shown in Fig. 2, fitted with an absorbed power-law, with absorbing column density fixed at $2.5 \times 10^{21} \mathrm{~cm}^{-2}$ (the expected interstellar absorption towards the globular cluster NGC 6712). The fit is a good deconvolution of the spectrum $\left(\chi^{2} /\right.$ d.o.f. $\left.=27.9 / 23\right)$ and results in a photon index of $2.2 \pm 0.2$ and in a $5-100 \mathrm{keV}$ flux corrected for the absorption of $(2.4 \pm 0.3) \times 10^{-10} \mathrm{erg} \mathrm{cm}^{-2} \mathrm{~s}^{-1}$ (based on the IBIS/ISGRI response matrix). Other simple models, like bremsstrahlung or a cut-off power-law, never result in a better fit.

4U 1850-087 has been observed with XMM-Newton on 27 September 2003 (see Sidoli et al. 2005), i.e. within the period covered by INTEGRAL data-set 2, with the main aim to study the low energy absorption intrinsic to the source. Indeed the XMM-Newton spectrum below $\sim 2 \mathrm{keV}$ is complex and we refer to Sidoli et al. 2005 for its detailed description (and for the XMM-Newton data reduction). EPIC PN operated in Small Window mode, and with a net exposure time of $8.1 \mathrm{ks}$. A combined spectral analysis EPIC/IBIS interestingly allows us to extend the spectral study of this source in the soft X-rays, below $5 \mathrm{keV}$, and to significantly refine the spectral parameters. During the XMM-Newton observation, only EPIC PN data did not suffer from pile-up problems, thus we use this spectrum $(1.7-12 \mathrm{keV}$, see Sidoli et al. 2005 for the details) to perform a broad-band analysis of the quasi-simultaneous EPIC-IBIS observations.

Among the different models considered by Sidoli et al. (2005) when fitting the XMM-Newton spectrum, we tried their best-fit, a disk-blackbody emission together with a power-law. 
Table 3. Results of the spectral analysis of the broad-band quasi-simultaneous spectrum (EPIC/PN-IBIS/ISGRI). Fluxes (2-100 keV) are corrected for the absorption, are in units of $10^{-10} \mathrm{erg} \mathrm{cm}^{-2} \mathrm{~s}^{-1}$, and are based on the EPIC pn response matrix. The assumed distance is $6.8 \mathrm{kpc}$ (Harris et al. 1996). The COMPTT model assumes a spherical geometry. $i$ is the inclination angle of the disk.

\begin{tabular}{lrlll}
\hline \hline Model & $\begin{array}{r}N_{\mathrm{H}} \\
\left(10^{22} \mathrm{~cm}^{-2}\right)\end{array}$ & Parameters & Flux & $\chi^{2} /$ d.o.f. \\
\hline DISKBB+POW & $0.4 \pm 0.2$ & $\Gamma=2.07_{-0.15}^{+0.07}$ & $2.8 \pm 0.1$ & $147.4 / 203$ \\
& & $k T_{\text {in }}=0.8 \pm 0.1 \mathrm{keV}$ & & \\
& & $r_{\text {in }} \times(\cos (i))^{0.5}=1.7_{-0.4}^{+1.1} \mathrm{~km}$ & & \\
\hline DISKBB+COMPTT & 0.25 fixed & $k T_{\mathrm{e}}>40 \mathrm{keV}$ & $2.9 \pm 0.1$ & $156.3 / 203$ \\
& & $k T_{0}=0.67 \pm 0.05 \mathrm{keV}$ & & \\
& & $\tau=2.2 \pm 0.5$ & \\
& $k T_{\text {in }}=k T_{0}$ & \\
& $r_{\text {in }} \times(\cos (i))^{0.5}=4 \pm 1 \mathrm{~km}$ & & \\
& &
\end{tabular}
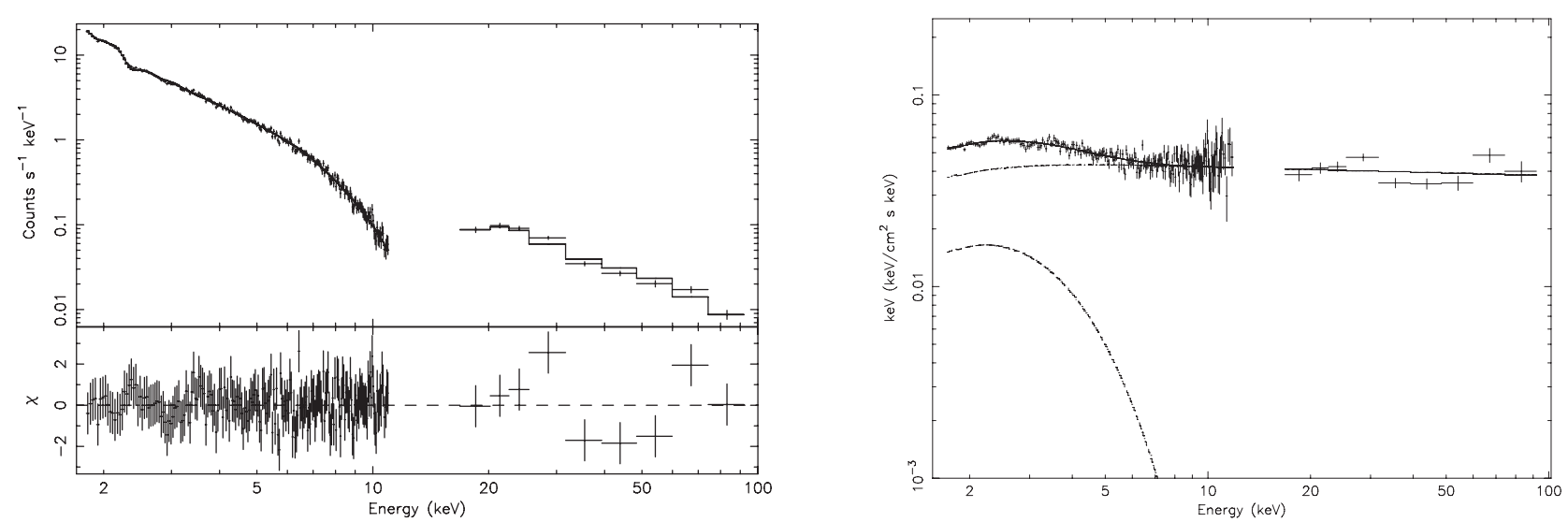

Fig. 3. Broad-band 4U 1850-087 EPIC/PN spectrum (obtained in September 2003) combined with the quasi-simultaneous IBIS/ISGRI (dataset 2). On the left, the count spectra are displayed, together with residuals in units of standard deviations, when fitted with a disk-blackbody plus a power-law. On the right, the corresponding $1.7-100 \mathrm{keV}$ energy spectrum is shown.

This gives already a very nice result $\left(\chi^{2} /\right.$ d.o.f. $\left.=147.4 / 203\right)$, with the spectral parameters reported in Table 3 and the spectrum shown in Fig. 3. The normalization constant for the IBIS/ISGRI spectrum relative to $\mathrm{PN}$ (constant factor fixed at 1) was $1.8_{-0.4}^{+0.2}$. We note that this normalization factor between the two instruments is in agreement with that reported for the Crab spectrum (Kirsch et al. 2005). The broad band flux (2-100 keV, corrected for the absorption) is $2.8 \times 10^{-10} \mathrm{erg} \mathrm{cm}^{-2} \mathrm{~s}^{-1}$ (based on the EPIC $\mathrm{PN}$ response matrix). Fixing the column density to the best-fit $\left(5.7 \times 10^{21} \mathrm{~cm}^{-2}\right)$ found by Sidoli et al. (2005), does not change the spectral results.

In order to obtain physical information, we then adopted the best-fit model used for the BeppoSAX 4U 1850-087 spectrum (Sidoli et al. 2001), a disk-blackbody plus a Comptonized emission (COMPTT model in XSPEC; Titarchuk 1994). Since the data extend only down to $1.7 \mathrm{keV}$, we linked the temperature of the seed photons, $k T_{0}$, to the inner disk temperature, $k T_{\text {in }}$. This is also justified by the empirical finding that in ultra-compact binaries the two temperatures are similar, within the uncertainties (Sidoli et al. 2001). We obtain a good fit $\left(\chi^{2} /\right.$ d.o.f. $\left.=156.3 / 203\right)$, with the parameters reported in Table 3 . To obtain better constraints to the spectral paramenters, in this case the absorbing column density has been fixed to the NGC 6712 interstellar absorption value. With this model, the normalization constant of the IBIS/ISGRI spectrum with respect to EPIC PN lies in the range 1.5-1.9, again in agreement with the standard value (Kirsch et al. 2005). Adopting a cut-off power-law instead of a power-law in the two-component model $\left(\chi^{2} /\right.$ d.o.f. $\left.=172.2 / 202\right)$, the cut-off energy resulted in $E_{\mathrm{c}}>110 \mathrm{keV}(90 \%$ confidence level), with a best-fit photon index of $1.9 \pm 0.1$.

\section{Discussion and conclusions}

Previously, the source broad-band spectrum was observed only with BeppoSAX in April 1997. At that time, the PDS nonimaging instrument, covering the range from 20 to $200 \mathrm{keV}$, detected the source only up to $50 \mathrm{keV}$ (Sidoli et al. 2001).

We report here for the first time the discovery of hard (50-100 keV) X-ray emission from the X-ray burster 4U 1850-087 and a long-term study of its X-ray spectral behaviour. It is now possible to directly measure the high energy luminosities of this source: they are $\sim 1.3 \times 10^{36} \mathrm{erg} \mathrm{s}^{-1}$ and $7 \times 10^{35} \mathrm{erg} \mathrm{s}^{-1}$ respectively in the $1-20 \mathrm{keV}$ and $20-200 \mathrm{keV}$ energy ranges (at $6.8 \mathrm{kpc}$ ). This implies that 4U 1850-087 falls into the so-called "burster box" (Barret et al. 2000).

In order to allow a proper comparison with the INTEGRAL spectrum we re-analysed the BeppoSAX observation (April 1997; refer to Sidoli et al. 2001 for the details of the data reduction) adopting the same model used here, a disk-blackbody emission together with a power-law. This resulted in the following parameters $\left(\chi^{2} /\right.$ d.o.f. $\left.=170.4 / 158\right)$ : an absorbing column density of $(0.46 \pm 0.03) \times 10^{22} \mathrm{~cm}^{-2}$, an inner disk blackbody temperature, $k T_{\text {in }}$, of $0.66 \pm 0.03 \mathrm{keV}$, and a powerlaw photon index of $1.96 \pm 0.06$. Thus, there is a good agreement with the INTEGRAL spectroscopy.

The new observations with INTEGRAL allow us to put much more stringent limits on the presence of a high energy cut-off. In Fig. 4 we compare the confidence contour levels for the high energy cut-off and the power-law photon index, obtained with the EPIC-IBIS joint spectrum (solid contours) and with BeppoSAX (dashed contours). During the BeppoSAX observation a lower 


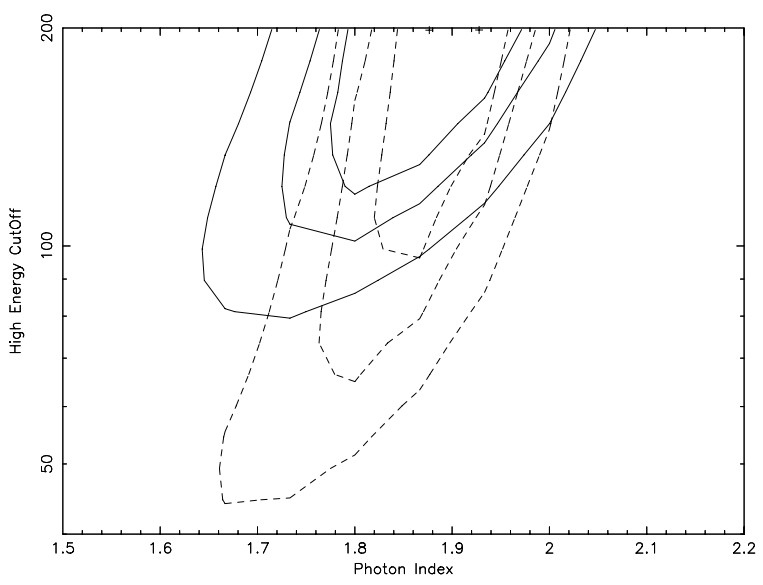

Fig. 4. Comparison of the confidence contour levels for the high energy cutoff (in units of $\mathrm{keV}$ ): solid contours have been derived analysing EPIC-IBIS joint spectrum, while the dashed contours mark the BeppoSAX results.

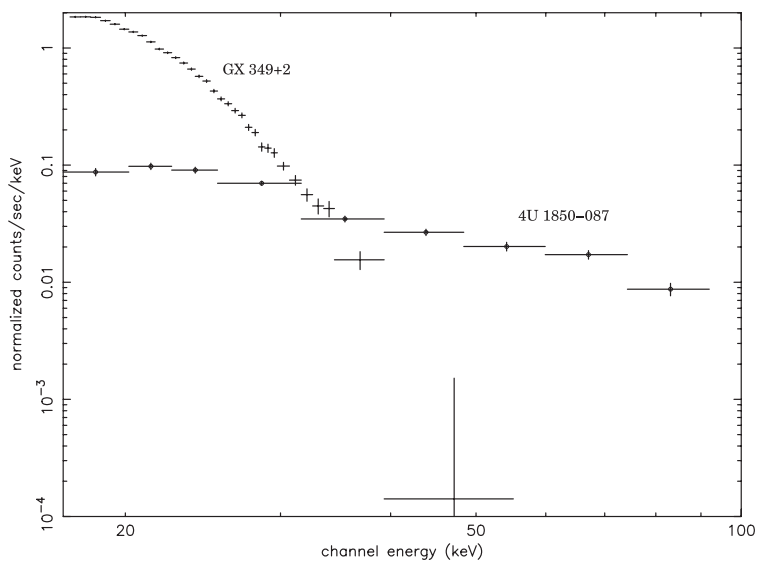

Fig. 5. Comparison of the INTEGRAL spectrum of the source 4U 1850-087 (data-set 2, the same dispayed in Fig. 3), with a spectrum of a typical Z-source, GX 349+2 (data from Paizis et al. 2006).

limit to the high energy cut-off could be placed at $E_{\mathrm{c}}>60 \mathrm{keV}$ (90\% level), while now with INTEGRAL we can interestingly shift it towards much higher energies, with $E_{\mathrm{c}}>100 \mathrm{keV}$. This allows us to include 4U 1850-087 among the few tens of lowluminosity low-mass X-ray binaries (LMXRBs) for which the spectrum has been observed up to $100 \mathrm{keV}$ (Barret et al. 2000; Di Salvo \& Stella 2002; Bazzano et al. 2006). More interestingly, $4 \mathrm{U} 1850-087$ is now among the hardest type I X-ray bursters in our Galaxy.

The INTEGRAL spectrum displays properties which fits well into the classification of low luminosity $\left(\sim 0.01 L_{\text {Edd }}\right)$ weakly magnetized neutron stars with "hard spectra" (Barret et al. 2001): a broad-band spectrum extending up to $100 \mathrm{keV}$, well described by a soft disk-blackbody emission together with a hard Comptonized component, with a optical depth of the Comptonizing corona of $\sim 2$, seed photons temperature below $1 \mathrm{keV}$, a high electron temperature, above $40 \mathrm{keV}$. A few LMXRBs appear to spend most of the time in this "hard state" (Di Salvo \& Stella 2002), like 4U 0614+091 (Piraino et al. 1999), which also displays spectral parameters similar to $4 \mathrm{U} 1850-087$, with a photon index in the range 2.3-2.4. Also for 4U 1850-087 the four different INTEGRAL spectra suggest that the source spend most of its lifetime in this spectral state (on timescales of months or years, if compared with BeppoSAX).

The very hard emission from $4 \mathrm{U} 1850-087$ is clearly evident also when compared (see Fig. 5) to a typical spectrum of a Z-source, GX 349+2, reported in an INTEGRAL study of the spectral behaviour of the persistent LMXRBs hosting a neutron star (Paizis et al. 2006).

Acknowledgements. We acknowledge the Italian Space Agency financial and programmatic support via contract I/R/046/04.

\section{References}

Anderson, S. F., Margon, B., Deutsch, E. W., \& Downes, R. A. 1993, AJ, 106, 1049

Barret, D. 2001, in Adv. Space Res., 28, 307

Barret, D., Olive, J. F., Boirin, L., et al. 2000, ApJ, 533, 329

Bazzano, A., et al. 2006, Proceedings of the conference, A life with stars, Amsterdam, 22-26 August 2005, New Astronomy Reviews, ed. Kaper, Van der Klis, \& Wijers, in press

Di Salvo, T., \& Stella, L. 2002, proceedings of the XXII Moriond Astrophysics Meeting, The Gamma-Ray Universe (Les Arcs, March 9-16, 2002), ed. A. Goldwurm, D. Neumann, \& J. Tran Thanh Van (Vietnam: The Gioi Publishers) [arXiv: astro-ph/0207219]

Harris, W. E. 1996, AJ, 112, 1487

Homer, L., Charles, P. A., Naylor, T., et al. 1996, MNRAS, 282, L37

Juett, A. M., \& Chakrabarty, D. 2005, ApJ, 627, 926

Juett, A. M., Psaltis, D., \& Chakrabarty, D. 2001, ApJ, 560, L59

Kirsch, M. G. F., Ubriel, U. G., Burrows, D., et al. 2005, SPIE, 5898, 22 [arXiv: astro-ph/0508235]

Lubinski, P., Dubath, P., \& Paltani, S. 2005, available at http://isdc. unige.ch/Soft/download/ osa/osa_doc/prod/osa_cross_cal-1.0.pdf

Labanti, C., Di Cocco, G., Ferro, G., et al. 2003, A\&A, 411, L149

Lebrun, F., Leray, J. P., Lavocat, P., et al. 2003, A\&A, 411, L141

Lund, N., Budtz-Jørgensen, C., Westergaard, et al. 2003, A\&A, 411, L231

Merloni, A., Fabian, A. C., \& Ross, R. R. 2000, MNRAS, 313, 193

Paltrinieri, B., Ferraro, F. R., Paresce, F., et al. 2001, AJ, 121, 3114

Paizis, A., Farinelli, R., Titarchuk, L., et al. 2006, A\&A, 559, 187

Parmar, A. N., Stella, L., \& Giommi, P. 1989, A\&A, 222, 96

Piraino, S., Santangelo, A., Ford, E. C., et al., 1999, A\&A, 349, L77

Sidoli, L., Parmar, A. N., Oosterbroek, T., et al. 2001, A\&A, 368, 451

Sidoli, L., La Palombara, N., Oosterbroek, T., \& Parmar, A. N. 2005, A\&A, 443, 223

Swank, J. H., Becker, R. H., Pravdo, S. H., et al. 1976, IAU Circ., 3010

Titarchuk, L. 1994, ApJ, 434, 570

Ubertini, P., Lebrun, F., Di Cocco, G., et al. 2003, A\&A, 411, L131

Vedrenne, G., Roques, J.-P., Schönfelder, V., et al. 2003, A\&A, 411, L63

Verbunt, F., Bunk, W., Hasinger, G., \& Johnston, H. M. 1995, A\&A, 298, 21 